\title{
O conhecimento sobre o câncer de mama e a mamografia das mulheres idosas frequentadoras de centros de convivência em São Paulo (SP, Brasil)
}

\author{
Awareness about breast cancer and mammography in elderly \\ women who frequent Daycare Centers in São Paulo (SP, Brazil)
}

Glenda Dias dos Santos ${ }^{1}$ Rosa Yuka Sato Chubaci ${ }^{1}$
${ }^{1}$ Curso de Graduação em Gerontologia, Escola de Artes, Ciências e

Humanidades,

Universidade de São Paulo. Av. Arlindo Béttio 1000, Ermelino Matarazzo. 03828-000 São Paulo SP. glendads@gmail.com
Abstract Early detection at the initial stage of breast cancer through mammography is the best way of reducing morbidity and mortality rates. The scope of this study was to establish awareness about breast cancer and mammography among elderly women, if they take the exam and discover the reasons that lead them to take the exam or not. A cross-sectional and exploratory study was conducted with 98 elderly women in three Senior Citizen Daycare Centers in São Paulo. With respect to awareness of mammography as the exam used for early detection of breast cancer, 55\% of the women answered that they knew. The majority of these women $(77.6 \%)$ had taken the exam and $22.4 \%$ had never done so. The reasons most frequently cited for taking a mammography were concern with health (53.8\%) and medical recommendation $(38.5 \%)$. The reasons for not taking the exam were not having a medical recommendation (50\%), conviction that they will never have the illness (23.1\%), didn't feel symptoms (19.2\%) and fear (7.7\%). This study showed that the subject of "breast cancer" is well known by the women, however mammography needs to be explained better. The fact that there are elderly women who have never submitted to the exam reveals the ongoing need to stage educational campaigns about breast cancer and the early detection exams.

Key words Beast cancer, Elderly women, Early detection, Mammography
Resumo A detecção precoce do câncer de mama em estágio inicial por meio da mamografia é das maneiras efetivas de se reduzirem as taxas de morbidade e mortalidade. Os objetivos deste artigo foram verificar o conhecimento que as mulheres idosas têm sobre o câncer de mama e mamografia; identificar se elas fazem esse exame e apontar os motivos que as levam ou não a realizarem esse exame. Trata-se de estudo exploratório de corte transversal, com 98 mulheres de três Centros de Convivência da Terceira Idade em São Paulo. Sobre o conhecimento da mamografia como exame usado na detecção precoce do câncer de mama, 55\% das mulheres responderam que conheciam. A grande maioria $(77,6 \%)$ costumava fazer esse exame, $e$ $22,4 \%$ nunca o fizeram. Os motivos mais apontados para se submeter à mamografia foram: preocupação com a saúde $(53,8 \%)$ erecomendação médica $(38,5 \%)$. Os motivos para não fazer o exame foram: não ter recomendação médica (50\%), nunca terá a doença (23,0\%), não teve sintomas (19,2\%) e medo (7,7\%). Este estudo mostrou que o assunto "câncer de mama" é bem conhecido pelas mulheres, porém a mamografia ainda precisa ser esclarecida quanto aos seus objetivos e recomendações. O fato de termos mulheres idosas que nunca realizaram oexame aponta a necessidade contínua de realizarmos ações educativas sobre o câncer de mama e seus exames de detecção precoce.

Palavras-chave Câncer de mama, Idosas, Detecção precoce, Mamografia 


\section{Introdução}

Nos últimos anos, países desenvolvidos e em desenvolvimento vêm enfrentando o envelhecimento de suas populações. O envelhecimento populacional é um fenômeno mundial que ocorre de maneira heterogênea entre esses países, cujo crescimento está relacionado com o aumento da expectativa de vida e o declínio das taxas de fecundidade e mortalidade ${ }^{1}$.

Muitos fatores influenciaram o processo do envelhecimento populacional no Brasil, tais como as mudanças na dinâmica da sociedade como a industrialização, urbanização, migração, modificações no estilo de vida, melhorias socioeconômicas, avanços tecnológicos e científicos. Assim como novas descobertas da medicina, que contribuíram para ampliar as medidas preventivas e mudar os padrões de morbimortalidade, diminuindo as doenças infecciosas e parasitárias e aumentando doenças crônico-degenerativas ${ }^{1}$.

Dessa maneira, nas últimas décadas, observou-se a redução da mortalidade na população brasileira e mudança no perfil das causas de morte entre os idosos, típico de uma população mais envelhecida. Entre as causas de morte, as neoplasias estão em terceiro lugar, sendo precedidas pelas doenças do aparelho circulatório e pelos sinais, sintomas e afecções mal definidas. A mortalidade por câncer, entre mulheres idosas, elevou-se de $10,5 \%$ em 1980 para $13,2 \%$ em $2000^{2}$.

Entre as neoplasias que mais acometem as mulheres idosas, o câncer de mama destaca-se como uma das principais causas de morte, fato que está relacionado a $60 \%$ dos casos serem descobertos tardiamente e à alta incidência dessa neoplasia com o avançar da idade ${ }^{3,4}$.

A detecção da doença em estágio inicial favorece tratamentos que podem erradicar totalmente o câncer de mama. Essa detecção precoce é realizada por meio do autoexame das mamas, exame clínico das mamas e a mamografia ${ }^{5,6,7}$. Dentre os métodos de detecção precoce, a mamografia é considerada a mais eficaz ${ }^{5,7-9}$.

A mamografia é uma radiografia da mama, que detecta lesões iniciais. Estudos mostram que a mamografia reduziu em média $30 \%$ dos óbitos de câncer em mulheres com mais de 50 anos $^{6}$. Este exame é indicado para mulheres assintomáticas, para rastreamento da neoplasia ${ }^{10}$; e para mulheres sintomáticas, para achados clínicos suspeitos de câncer mamário ${ }^{11}$.

A mamografia detecta de $80 \%$ a $90 \%$ dos casos de câncer de mama em mulheres assintomáticas $^{12}$. Isso tem ajudado a reduzir a mortalidade por câncer de mama, pois este exame permite a antecipação, em cerca de dois anos, do diagnóstico. Dessa maneira, a mamografia permite que a doença seja detectada precocemente, colaborando com um tratamento mais eficiente, menor dano estético e diminuição da morbidade ${ }^{13}$.

No Brasil, o Instituto Nacional de Câncer (Inca) sugere que a mamografia seja realizada, no máximo, a cada dois anos em mulheres de 50 a 69 anos. Em mulheres com caso de câncer de mama na família, a mamografia e o exame clínico das mamas devem ser realizados anualmente, a partir dos 35 anos de idade ${ }^{14}$.

No entanto, o Projeto Diretrizes, da Associação Médica Brasileira e Conselho Federal de Medicina, que procurou englobar informações da área médica sobre o rastreamento mamográfico, acrescentou na recomendação do Inca que mulheres entre 40 e 49 anos façam anualmente a mamografia, e nas mulheres acima dos 70 anos a decisão deverá ser individual, considerando a expectativa de vida de cada mulher, pois a incidência do câncer de mama aumenta com a idade ${ }^{15}$. A incidência da doença aos 40 anos é um caso para 232 mulheres, e aos 70 anos é um para $29^{8}$.

Com base nessas considerações, verificou-se a importância da detecção precoce do câncer de mama na terceira idade, pois a doença detectada em estágio inicial aumenta a probabilidade de cura, evitando sofrimento e a mortalidade. Portanto, os objetivos do presente estudo são: verificar o conhecimento que as mulheres idosas têm sobre o câncer de mama e mamografia; e apontar os motivos que levam ou não as participantes do estudo a realizarem ou não a mamografia.

\section{Método}

Realizou-se um estudo exploratório de corte transversal, com 98 mulheres idosas de três Centros de Convivência localizados na zona Leste da cidade de São Paulo. Fizeram parte do estudo as mulheres idosas moradoras há mais de um ano na região e que aceitaram participar do estudo mediante esclarecimento prévio e assinatura do Termo de Consentimento Livre e Esclarecido, conforme Resolução CNS nº 196/96-MS. Este projeto foi aprovado pelo Comitê de Ética e Pesquisa da Escola de Enfermagem da Universidade de São Paulo.

Utilizou-se a técnica de amostragem aleatória estratificada, e para a coleta dos dados (realizado no Centro de Convivência) foi empregada a técnica da entrevista, sendo utilizado como ins- 
trumento um formulário semiestruturado. Para participar do estudo, foram sorteadas $154 \mathrm{mu}$ lheres, incluindo $30 \%$ das perdas; não conseguimos entrar em contato com 31 mulheres; 13 se recusaram a participar do estudo; 12 faltaram na entrevista; no final, apenas 98 responderam ao formulário.

Esse formulário constava de dados de identificação e perguntas sobre o conhecimento em relação ao câncer de mama e à mamografia, a realização da mamografia, dificuldades em relação ao exame e os motivos que levam a fazer ou não a mamografia.

Os dados foram tabulados no Excel e analisados no programa SPSS. Para a análise dos dados foi adotada a distribuição das frequências absolutas e relativas, e utilizadas medidas de associação (coeficientes de contingência Phi e V de Crámer) em: costuma fazer mamografia e onde realizou o exame; costuma fazer mamografia e conhece os sintomas do câncer de mama; costuma fazer a mamografia e conhece os exames. O nível de significância adotado foi de 5\%. Considerou-se como significante quando o valor de $\mathrm{p}$ foi inferior a 0,05 .

\section{Resultados e discussão}

Em relação aos dados de identificação das participantes, a média de idade foi de 71,3 anos. A grande maioria das mulheres - 74 (75,5\%) - possuía o ensino fundamental incompleto, $76(77,5 \%)$ tinham renda de um a dois salários mínimos e 78 $(79,6 \%)$ informaram que não tinham convênio médico particular e quando necessário utilizavam os serviços públicos de saúde.

Todas as mulheres do estudo já escutaram falar do câncer de mama. Dentre as respostas sobre onde obtiveram as informações sobre essa doença, 53 (31,9\%) referiam-se aos meios de comunicação, $34(20,5 \%)$ a palestras, $27(16,3 \%)$ a amigos, $21(12,7 \%)$ à família e $31(18,7 \%)$ aos profissionais de saúde. Em relação à informação sobre a mamografia, 14 (10,9\%) referiram ter obtido informação em meios de comunicação, $15(11,7 \%)$ em palestras, 7 (5,5\%) com amigos, 7 (5,5\%) na família, $38(29,7 \%)$ com profissionais de saúde e $47(36,7 \%)$ referiram não terem recebido informação sobre o exame, conforme mostra a Tabela 1.

Esses resultados mostram que os meios de comunicação são uma das principais fontes de informação para o câncer de mama, indo ao encontro do estudo de Godinho e Koch ${ }^{11}$, no qual a televisão destacou-se nas respostas sobre as fontes utilizadas para se obter conhecimento do câncer de mama, seguida pelas revistas, conversas interpessoais, médico, jornais, rádio e Internet ${ }^{11}$.

Quanto à mamografia, os profissionais de saúde são citados como as principais fontes de informação sobre o exame. Já no trabalho de Vieira e Koch ${ }^{12}$, a maioria das mulheres entrevistadas referiu que recebeu informação sobre a mamografia com os médicos, em seguida com pessoas leigas e os meios de informação.

Um fato que não pode deixar de ser citado é que neste estudo uma parcela significativa não recebeu informação sobre a mamografia, resultado também encontrado no estudo de Marchi ${ }^{13}$.

O conhecimento sobre os sintomas do câncer de mama contribui para um diagnóstico precoce $^{16}$. Com relação ao conhecimento sobre os sintomas do câncer de mama, dentre as entrevistadas, $70(71,4 \%)$ responderam que conhecem algum sintoma do câncer de mama e 28 (28,6\%) não conhecem. Das mulheres que conhecem os sintomas, $50(71,4 \%)$ referiram presença de caroço ou nódulo, $3(4,3 \%)$ dor no seio, $6(8,6 \%)$ caroço e dor, 2 (2,9\%) caroço e sangue ou líqui-

Tabela 1. Distribuição das respostas referidas pelas mulheres, segundo a fonte de informação sobre o câncer de mama e a mamografia - São Paulo (SP), 2007.

\begin{tabular}{lrr}
\hline \multicolumn{1}{c}{ Fontes de informação } & Câncer de mama & \multicolumn{1}{c}{ Mamografia } \\
\hline Meios de comunicação & $53(31,9 \%)$ & $14(10,9 \%)$ \\
Palestras & $34(20,5 \%)$ & $15(11,7 \%)$ \\
Amigos & $27(16,3 \%)$ & $7(5,5 \%)$ \\
Família & $21(12,6 \%)$ & $7(5,5 \%)$ \\
Profissionais de saúde & $31(18,7 \%)$ & $38(29,7 \%)$ \\
Não recebeu informação & $0(0,0 \%)$ & $47(36,7 \%)$ \\
Total & $\mathbf{1 6 6}(\mathbf{1 0 0 , 0} \%)^{*}$ & $128(100,0 \%)^{*}$
\end{tabular}

*Algumas mulheres mencionaram mais que uma fonte de informação. 
do no bico, $4(5,7 \%)$ caroço e ferida ou mancha e $5(7,1 \%)$ outras respostas, tais como: glândula, tosse e ferimento no corpo, pancada ou batida que não é cuidada, inflamação da mama, caroço acompanhado de coceira.

Em outros estudos, como de Linsell et al. ${ }^{17}$, encontrou-se um número considerável de mulheres idosas que não tinham o costume de verificar os seus seios. Todavia, a grande maioria tinha o conhecimento que um nódulo na mama ou na axila poderia ser um sintoma do câncer de mama.

Esses dados apontam a necessidade de ações educativas sobre os sintomas do câncer de mama, pois verifica-se que $7,1 \%$ têm conhecimentos que precisam ser mais bem esclarecidos. $\mathrm{O}$ fato de a presença do caroço ou nódulo ter sido referida pela grande maioria $(71,4 \%)$ pode estar relacionado com as campanhas educativas sobre o autoexame presentes na mídia, sendo a mensagem mais referida pelas mulheres apalpar os seios em busca de caroço Linard et al. ${ }^{18}$.

Nesse sentido, Castro ${ }^{19}$ menciona que, nos últimos anos, a mídia aumentou o número de matérias relacionadas à detecção precoce, principalmente sobre o câncer de mama e a importância da realização de seus exames de detecção precoce (mamografia, ultrassonografia e autoexame).

A respeito do conhecimento sobre algum exame utilizado para descobrir o câncer de mama (detecção precoce), verificou-se que 22 (22,4\%) não conheciam nenhum exame para detectar o câncer de mama, $76(77,6 \%)$ conheciam algum exame, entretanto $38(38,8 \%)$ não conseguiam expressar o nome correto do exame, referindose aos exames pelas características, como "aquele exame que aperta o seio" (mamografia), "o médico apalpa" (exame clínico das mamas) e "a massagem do seio ou apalpação” (autoexame), por exemplo.

Nesse contexto, algo a ser destacado é o fato de $22,4 \%$ das mulheres responderem que não conheciam nenhum exame para detectar o câncer de mama, o que pode estar correlacionado à ausência ou diminuição da frequência em consultas ginecológicas e, consequentemente, à redução ou não realização desses exames, pois com o avançar da idade as mulheres idosas, quando comparadas com as mais jovens, reduzem os cuidados de saúde preventivos ${ }^{20}$.

Os dados da Tabela 2 demonstram as respostas das participantes sobre os exames utilizados na detecção precoce do câncer de mama. Dentre as respostas, $61(55,0 \%)$ citaram a mamografia, $33(29,7 \%)$ o autoexame, 11 (9,9\%) o exame clínico das mamas e $6(5,4 \%)$ a ultrasso- nografia das mamas (exame complementar à mamografia).

Observa-se que, dos exames utilizados na detecção precoce, a mamografia foi a mais referida por $55 \%$ mulheres. Este fato pode estar relacionado à idade, pois $46 \%$ estão entre 50 e 69 anos, faixa etária na qual o Ministério da Saúde preconiza o rastreamento mamográfico ${ }^{14}$. Por sua vez, as atitudes relacionadas ao corpo, como o autoexame, incluem fatores culturais, tabus, valores e crenças para a mulher ${ }^{9}$, que podem estar envolvido no fato de não ter sido muito citado pelas entrevistadas.

Já o exame clínico das mamas foi menos referido do que os anteriores, talvez porque a técnica não esteja sendo realizada periodicamente nessas mulheres. Em estudo realizado no município de Botucatu, verificou-se que as mulheres mais jovens eram mais examinadas nas mamas do que as mais velhas ${ }^{21}$, apontando para a necessidade de programas de educação em saúde e reformulação das campanhas educativas que talvez poderiam abordar a detecção precoce do câncer de mama, por meio de uma linguagem mais próxima do universo da mulher idosa.

Das mulheres do estudo, $76(77,6 \%)$ costumavam fazer a mamografia e $22(22,4 \%)$ nunca se submeteram ao exame. Dessas mulheres que costumavam fazer a mamografia, 39 (51,3\%) realizavam anualmente, $6(7,9 \%)$ de 2 em 2 anos, $19(25,0 \%)$ esporadicamente e 12 (15,8\%) só realizou uma vez. Estes dados contrapõem-se aos achados de Lima Costa e Matos ${ }^{22}$ no que diz respeito à realização da mamografia pelas mulheres idosas, em que 54\% nunca realizaram e $46 \%$ já haviam se submetido ao exame.

O conhecimento é fator fundamental para realizar a mamografia. Verifica-se que há associação significativa $(p=0,047)$ entre: costuma fazer

Tabela 2. Distribuição das respostas referidas pelas mulheres, segundo os exames utilizados na detecção precoce do câncer de mama - São Paulo (SP), 2007.

\begin{tabular}{lrr}
\hline $\begin{array}{l}\text { Exames utilizados na detecção } \\
\text { precoce do câncer de mama }\end{array}$ & n & \multicolumn{1}{c}{$\%$} \\
\hline Autoexame das mamas & 33 & 29,7 \\
Exame clínico das mamas & 11 & 9,9 \\
Mamografia & 61 & 55,0 \\
Ultrassonografia & 6 & 5,4 \\
Total & $\mathbf{1 1}^{*}$ & $\mathbf{1 0 0 , 0}$ \\
\hline
\end{tabular}

"Algumas mulheres mencionaram mais de um exame. 
mamografia e conhece os sintomas do câncer de mama, costuma fazer a mamografia e conhece os exames $(p=0,012)$.

Conforme Rutledge et al. ${ }^{23}$, o aumento do conhecimento sobre o câncer de mama e de seus exames de detecção precoce aumentam a motivação em relação à saúde, podendo influenciar a prática da realização desses exames.

Para Linsell et al. ${ }^{17}$, o aumento da sensibilização sobre o câncer de mama pode promover a sua detecção precoce, principalmente na mulher idosa, que tem mais riscos para desenvolvê-lo e demora mais para reconhecer os sintomas e procurar auxílio do que as mulheres jovens.

Neste estudo, a grande maioria das mulheres - $57(75 \%)$ - realizou a mamografia entre menos de um ano a dois anos e $25 \%$ há três anos ou mais, o que mostra que a grande maioria está em dia com o exame. Estes dados vão ao encontro dos achados de Vieira e Koch ${ }^{12}$, em que 71,2\% das mulheres realizaram a mamografia entre menos de um ano a dois anos e 28,8\% há três anos ou mais.

Com relação ao local da realização da última mamografia, este estudo verificou que 53 (69,7\%) mulheres relataram ter realizado pelo Sistema Único de Saúde (SUS), 21 (27,6\%) pelo convênio e $2(2,6 \%)$ de forma particular. Verifica-se que há associação significante $(\mathrm{p}=0,001)$ entre: costuma fazer mamografia e onde realizou - demonstrando que o fato de ter ou não um plano de saúde ou ter feito o exame de forma particular não influencia as mulheres deste estudo a realizarem ou não a mamografia. Isso talvez possa ser explicado pelo fato de que na cidade de São Paulo há diversos recursos de saúde disponíveis para a população.

No entanto, pesquisadores constataram diferenças no acesso aos serviços de saúde na cidade de Taubaté (SP). As mulheres que realizaram a mamografia pelo convênio médico ou particular realizaram mais vezes o exame do que as que fizeram pelo SUS ${ }^{24}$.
Das 76 mulheres do estudo que fizeram a mamografia, $33(43,4 \%)$ referiram ter dificuldades e 43 (56,6\%) não. As dificuldades citadas foram: achava o exame dolorido - 27 (27,6\%); havia demora para marcar o exame - $10(10,2 \%)$; sente vergonha $-7(7,1 \%)$; demora para fazer o exame - 5 (5,1\%); medo do exame - $5(5,1 \%)$; mal atendimento no momento do exame - 2 (2\%); e receio de descobrir algo - $2(2 \%)$.

Freitas Jr. et al..$^{25}$ mencionam que o desconforto e a dor são frequentemente referidos pelas mulheres durante a realização da mamografia, sendo que mulheres com menos de 50 anos apresentavam mastalgia prévia e queixavam-se mais do que as idosas.

Considerando essas informações, algumas mulheres que apresentaram dificuldades na realização da mamografia poderiam prolongar o intervalo para se submeter novamente ao exame. Neste estudo, não encontramos associação significante entre dificuldade e intervalo para realizar a mamografia $(p=0,572)$.

Dentre as participantes do estudo, 79,6\% eram usuárias dos serviços públicos e $69,7 \%$ costumavam realizar a mamografia pelo SUS. Destas que fizeram o exame, 43,4\% mulheres referiram sentir dificuldade. Este dado sugere que o fato de sentir dificuldades para realizar a mamografia não foi um empecilho para as mulheres deste estudo realizarem o exame.

Entre os motivos para fazer a mamografia, contidos na Tabela 3, as mulheres referiram principalmente a preocupação com a saúde (53,8\%). Algumas fizeram o exame porque estavam preocupadas com dor ou caroço que sentiram no seio, outras porque o exame serve para saber se têm câncer ou outra doença, porque o exame é importante para a prevenção do câncer de mama e por gostar de cuidar da saúde.

Destacou-se, também, recomendação médica $(38,5 \%)$. Algumas fazem a mamografia por ser um exame de rotina e outras fazem por obri-

Tabela 3. Distribuição das respostas referidas pelas mulheres, segundo os motivos para realizar ou não a mamografia - São Paulo (SP), 2007.

\begin{tabular}{lrlr}
\hline \multicolumn{1}{c}{ Motivos para realizar a mamografia } & \multicolumn{1}{c}{$\mathbf{n}(\%)$} & Motivos para não realizar & \multicolumn{1}{c}{$\mathbf{n}(\%)$} \\
\hline Preocupação com a saúde & $42(53,8 \%)$ & Não teve recomendação médica & $13(50,0 \%)$ \\
Recomendação médica & $30(38,5 \%)$ & Nunca terá a doença & $6(23,1 \%)$ \\
Medo da doença & $5(6,4 \%)$ & Não teve sintomas & $5(19,2 \%)$ \\
História na família & $1(1,3 \%)$ & Medo & $2(7,7 \%)$ \\
Total & $78(100 \%)$ & Total & $26(100 \%)$ \\
\hline
\end{tabular}


gação. O medo da doença $(6,4 \%)$ está presente entre os motivos para se fazer o exame. Algumas mulheres referiram que conheciam pessoas que tiveram câncer de mama e tinham medo de serem acometidas pela doença.

Os achados de Rutledge et al. ${ }^{23}$ revelam que os motivos para as mulheres realizarem a mamografia estavam relacionados com: a idade (ter mais de 50 anos), incentivo médico ou da enfermeira e maior risco para o câncer de mama.

De acordo com Molina et al. ${ }^{21}$, as mulheres estão cada vez mais preocupadas com o câncer de mama devido à alta incidência dessa neoplasia e às campanhas educativas presentes nos meios de comunicação, o que estaria levando essas mulheres a procurarem os métodos de prevenção.

Para algumas mulheres, o fato de ter história de câncer de mama na família $(1,3 \%)$ fez com que elas realizassem esse exame, pois ouviram que pessoas que têm história familiar de parentes do primeiro grau acometidos pela doença tinham risco elevado de desenvolvê-la.

As mulheres do estudo que não realizaram a mamografia referiram os seguintes motivos: não teve recomendação médica, acreditava que nunca terá a doença, não ter sintomas da doença e medo do exame. Estes dados corroboram os achados de Black et al. ${ }^{26}$, nos quais os motivos para as mulheres idosas nunca terem se submetido à mamografia eram porque "o médico não recomendou" e "acha que não precisa fazer".

A falta de recomendação médica foi um dos principais motivos referidos (por 50\% das mulheres) para não realizar a mamografia. Esse dado também foi encontrado em outros estudos, alertando que o fato de não ter sido recomendada pode estar relacionado à ausência de consultas médicas ou ter sido recomendada pelo médico e não ter feito o exame $e^{11,13,21}$.

Muitas mulheres do estudo acreditavam que não necessitavam fazer o exame por não terem sintomas da doença (19,2\%), como por exemplo a presença de caroços nos seios, pois as campanhas educativas sobre o câncer de mama que são exibidas nos canais de televisão destacam a importância da apalpação dos seios para encontrar caroços. Como elas não encontram caroço ou não observam nenhuma anormalidade, podem estar entendendo que não há necessidade da mamografia e que nunca terão a doença $(23,1 \%)$.

Algumas mulheres referiram sentir medo do exame $(7,7 \%)$, porque consideraram um procedimento muito dolorido. Este fato pode ser justificado em razão de a mamografia ter características de ser incômoda na sua realização e para algumas mulheres ela ser dolorosa ${ }^{25}$. O estudo de Marchi $^{13}$ também constatou que o medo era um dos motivos para mulheres usuárias do serviço público não realizarem a mamografia.

\section{Conclusão}

O assunto "câncer de mama" é bem conhecido pelas mulheres, porém a mamografia ainda precisa ser esclarecida quanto aos seus objetivos e recomendações. O conhecimento sobre os sintomas e os exames utilizados na detecção precoce do câncer de mama influenciou as mulheres do estudo a realizarem a mamografia, mostrandonos a importância da conscientização sobre essa doença.

Os dados encontrados neste estudo reforçam a necessidade de implementação de ações educativas para as mulheres idosas que esclareçam a importância do autoexame e do exame clínico das mamas, que foram pouco referidos pelas participantes. $\mathrm{O}$ fato de termos mulheres idosas que nunca realizaram o exame aponta a necessidade contínua de realizarmos ações educativas sobre o câncer de mama e seu exame de detecção precoce.

Perante os motivos apresentados para realização do exame, o estudo mostrou-nos que a questão subjetiva sobre a importância da detecção sobrepõe as dificuldades encontradas para realizar a mamografia.

Os motivos alegados pelas mulheres para não realizarem os exames de detecção precoce do câncer de mama são pontos importantíssimos a serem repensados pelos profissionais de saúde que atuam na área. Os resultados deste estudo mostraram que ainda existem, em proporção menor, mulheres que não se submetem ao exame. Dessa forma, garantir a adesão da mulher a esse cuidado preventivo continua sendo preocupante e desafiador.

A ação educativa com respeito mútuo cliente-profissional de saúde é um importante passo para que a mulher compreenda a importância do exame preventivo e sinta-se motivada a realizá-lo. Esse relacionamento interpessoal, por meio de uma comunicação efetiva, é primordial para que a mulher tenha confiança no profissional de saúde e supere as eventuais dificuldades, como as identificadas neste estudo.

DesSa forma, é fundamental que o papel do profissional de saúde com as mulheres idosas seja orientá-las quanto à frequência das consultas ginecológicas e quanto à importância em realizar periodicamente exames de detecção precoce 
como a mamografia, o exame clínico das mamas e o autoexame, pois grande parte das mulheres ao fim da menopausa acredita que não precisam mais de um cuidado ginecológico. É importante enfatizar que não há uma idade limite para o cuidado ginecológico e também informar que deve ser contínuo, variando de intervalo conforme a história clínica de cada mulher.

É importante que novas pesquisas sejam realizadas com o tema detecção precoce do câncer de mama em mulheres idosas. Nosso estudo tem a limitação de envolver apenas três Centros de Convivências da Zona Leste da cidade de São Paulo, porém consideramos como mais uma contribuição para as pesquisas na área da gerontologia e um estímulo para o desenvolvimento de futuros trabalhos na área da prevenção na terceira idade.

\section{Colaboradores}

GD Santos e RYS Chubaci trabalharam conjuntamente na concepção teórica, coleta e análise dos dados, discussão dos resultados, elaboração e redação final do texto.

\section{Agradecimentos}

Ao Programa Institucional de Bolsas de Iniciação Científica da Universidade de São Paulo, pelo apoio financeiro recebido para a realização da pesquisa; aos dirigentes e pessoas idosas dos Centros de Convivência participantes da pesquisa. 


\section{Referências}

1. Paschoal SMP, Salles RFN, Franco RP. Epidemiologia do envelhecimento. In: Carvalho Filho ET, Papaléo Netto M, organizadores. Geriatria: fundamentos, clínica e terapêutica. $2^{\text {a }}$ ed. São Paulo: Atheneu; 2005. p. 19-23.

2. Camarano AA, Kanso S, Mello JL. Como vive o idoso brasileiro? In: Camarano AA, organizador. Os novos idosos brasileiros: muito além dos 60? Rio de Janeiro: Ipea; 2004. p. 25-73.

3. Carvalho Filho ET, Klein EL, Soares RR. Medicina preventiva. In: Carvalho Filho ET, Papaléo Netto M, organizadores. Geriatria: fundamentos, clínica e terapêutica. 2a ed. São Paulo: Atheneu; 2005. p. 687-698.

4. Chaimowicz F. Epidemiologia e o envelhecimento no Brasil. In: Freitas EV, Py L, Cançado FAX, Gorzoni ML, organizadores. Tratado de geriatria e gerontologia. $2^{a}$ ed. Rio de Janeiro: Guanabara Koogan; 2006. p. 106-130.

5. Bergamasco RB, Tsunechiro MA. Câncer de mama e auto-exame. In: Fernandes RAQ, Narchi NZ, organizadores. Enfermagem e saúde da mulher. São Paulo: Manole; 2007. p. 116-121.

6. Brasil. Secretaria de Atenção à Saúde. Departamento de Atenção Básica. Controle dos cânceres do colo do útero e da mama. Brasília: Ministério da Saúde; 2006.

7. Otto SE. Câncer mamário. In: Otto SE. Oncologia. Rio de Janeiro: Reichmann \& Affonso Editores; 2002. p. 105-119.

8. Brasil. Ministério da Saúde. Secretaria de Atenção à Saúde. Departamento de Atenção Básica. Envelhecimento e saúde da pessoa idosa. Brasília: Ministério da Saúde; 2006.

9. Pinotti JA, Nisida ACT. Detecção precoce e prevenção. In: Lima GR, Gebrim LH, Oliveira VC, Martins NV, organizadores. Ginecologia oncológica. São Paulo: Atheneu; 1999. p. 34-41.

10. Instituto Nacional de Câncer (Inca). Encontro Internacional sobre Rastreamento de Câncer de Mama. Rev Bras de Cancerol 2009; 55(2):99-113.

11. Godinho ER, Koch HA. O perfil da mulher que se submete a mamografia em Goiânia: uma contribuição a bases para um programa de detecção precoce do câncer de mama. Radiol Bras 2002; 35(3): 139-145.

12. Vieira AV, Koch HA. Conhecimento sobre mamografia por mulheres que freqüentam o serviço de radiologia da Santa Casa de Misericórdia do Rio de Janeiro (SCMRJ). Rev Bras de Mastologia 1999; 9:56-61.

13. Marchi AA. Rastreamento do câncer mamário: características de utilização da mamografia em serviços de saúde públicos e privados [dissertação]. Campinas: Universidade Estadual de Campinas; 2004.

14. Instituto Nacional de Câncer (Inca). Controle do câncer de mama: documento do consenso. Rio de Janeiro: Inca; 2004.

15. Kemp C, Petti DA, Ferraro O, Elias S. Câncer de mama: prevenção secundária. Sociedade Brasileira de Mastologia, Federação Brasileira das Sociedades de Ginecologia e Obstetrícia. Projeto Diretrizes. São Paulo: Associação Médica Brasileira, Conselho Federal de Medicina; 2002.
16. Gebrim LH, Facina G, Navarrete MALH, Nazário ACP, Kemp C, Lima GR. Aspectos clínicos e terapêuticos do carcinoma de mama em pacientes idosas: estudo de 72 casos. Rev Bras Ginecol Obstet 1995; 17(2):931-938.

17. Linsell L, Burgess CC, Ramirez AJ. Breast cancer awareness among older women. British Journal of Cancer 2008; 99(8):1221-1225.

18. Linard AG, Amorim FC, Machado FAS. Detecção precoce do câncer de mama: os efeitos da comunicação de mama na opinião de mulheres. In: Carvalho EC, organizador. Comunicação em enfermagem: relatos de pesquisas do $6^{\circ}$ Simpósio Brasileiro de comunicação em enfermagem. Ribeirão Preto, SP: Escola de Enfermagem de Ribeirão Preto, Fundação Instituto de Enfermagem de Ribeirão Preto; 1998.

19. Castro R. Câncer na mídia: uma questão de saúde pública. Rev Brasileira de Cancerologia 2009; 55(1):4149 .

20. Sclowitz ML, Menezes AMB, Gigante DP, Tessaro S. Condutas na prevenção secundária do câncer de mama e fatores associados. Rev Saude Publica 39(3):340-349.

21. Molina L, Dalben I, De Luca LA. Análise das oportunidades de diagnóstico precoce para as neoplasias malignas de mama. Rev Assoc Med Bras 2003; 49(2):185-190.

22. Lima Costa MF, Matos DL. Prevalência e fatores associados à realização da mamografia na faixa etária de 50-69 anos: um estudo baseado na Pesquisa Nacional por Amostra de Domicílios (2003). Cad Saude Publica 2007; 23(7):1665-1673.

23. Rutledge, DN, Barsevick M, Knobif MT, Bookbinder M. Breast cancer detection: knowledge, attitudes, and behaviors of women from Pennsylvania. Oncology Nursing Forum 2001; 28(6):1032-1040.

24. Marchi AA, Gurgel MSC, Fonsechi-Carvasan GA. Rastreamento mamográfico do câncer de mama em serviços de saúde públicos e privados. Rev Bras Ginecol Obstet 2006; 28(4):214-219.

25. Freitas Jr R, Fiori WF, Ramos FJF, Godinho E, Rahal RMS, Oliveira JG. Desconforto e dor durante realização da mamografia. Rev Assoc Med Bras 1992; 52(5):333-336.

26. Black MEA, Stein KF, Loveland-Cherry CJ. Older women and mammography screening bhavior: do possible selves contribute? Health Education \& Behavior 2001; 28(2):200-216.

Artigo apresentado em 15/05/2009

Aprovado em 17/06/2009

Versão final apresentada em 17/07/2009 